\title{
FRA CONTEMPLAZIONE E DOMINIO NOTE IN MARGINE AL RAPPORTO UOMO-NATURA NEL PENSIERO MEDIEVALE
}

\author{
Gregorio Piaia*
}

SÍNTESE - As relações entre homem e natureza, no pensamento medieval, podem ser examinadas por diversos ângulos e por todos eles leva-se em consideração a Deus, como criador. As posições extremas do homem ante a natureza situam-se entre a contemplação e o domínio. Dois pensadores são aqui examinados: Hugo de São Víctor e Petrarca.

PALAVRAS-CHAVE - Filosofia medieval. Hugo de São Víctor. Petrarca. Homem. Natureza. Deus.
ABSTRACT - Relationships between man and nature in medieval thought can be examined from different standpoints, as they all take God qua creator into account. The extreme positions of man towards nature can be placed between contemplation and dominion. Two thinkers are considered here: Hugus of Saint Victor and Petrarca.

KEY WORDS - Hugus of Saint Victor. Petrarca. Man. Nature. God.

Impossibile, nel giro di poche pagine, mettere a fuoco il tema "uomo e natura nel pensiero medievale", tanto più che si tratta in realtà di un rapporto a tre, che coinvolge necessariamente - nella comune coscienza dell'età di mezzo - la relazione con la figura di Dio creatore (il che, sul piano speculativo, non è cosa di poco conto). D'altronde il tema si presta a una varietà di approcci, a seconda che il rapporto uomo-natura sia inteso nel senso di una definizione del concetto stesso di 'natura' ("La natura è una forza insita nelle cose che, da cose simili, produce cose simili": così scriveva Guglielmo di Conches alla fine del I libro del Dragmaticon philosophiae) ${ }^{1}$ oppure di un'autonoma e sistematica conoscenza della natura da parte dell'umano intelletto (all'insegna del motto albertino de naturalibus naturaliter) oppure dell'intrinseca costituzione dell'uomo stesso (e quindi, per usare termini di conio più recente, dell'“antropologia filosofica"). ${ }^{2}$ S'impone allora

* Università di Padova.

1 TEODORICO di CHARTRES, GUGLIELMO di CONCHES, BERNARDO SILVESTRE, II divino e il megacosmo. Testi filosofici e scientifici della scuola di Chartres, a cura di E. Maccagnolo, Milano 1980, p. 262. Ma si veda in proposito T. GREGORY, L'idea di natura nella filosofia medievale prima dell'ingresso della fisica di Aristotele, in La filosofia della natura nel medioevo. Atti del III Congresso internazionale di filosofia medievale, Milano 1966, p. 27-65.

2 Cfr., ad es., il saggio di S. VANNI ROVIGHI, Uomo e natura. Appunti per una antropologia filosofica, Milano 1980, che, pur muovendo da un impianto fenomenologico e da un'analisi dei modemi "maestri del sospetto", ripropone in forma aggiornata il procedimento aristotelico-tomistico della

ก. 3

Setembro 1999

p. $843-851$ 
una radicale delimitazione del campo e delle modalità di approccio: il nostro intento è d'illustrare due posizioni-tipo per quel che attiene al modo con cui nel medioevo gl'"intellettuali" (ci si perdoni questo evidente anacronismo terminologico) guardavano alla "natura" e si rapportavano ad essa. E' una scelta del tutto arbitraria e riduttiva, ed anche alquanto provocatoria, perché non concerne quelli che sono solitamente considerati i massimi esponenti del pensiero medievale; una scelta che non pretende quindi di offrire una panoramica d'insieme sulle concezioni del medioevo intorno all'uomo e alla natura, ma che, facendo leva su due exempla a nostro avviso particolarmente significativi, è però in grado di farci cogliere dal vivo una mutazione culturale che ha aperto la via alla "modernità". In tale modo è altresì possibile trarre qualche spunto di riflessione in ordine al problema - dominante in questi anni del "postmoderno" e ancor più nel prossimo millennio - di un più equilibrato rapporto fra uomo e natura.

Il primo autore che vorremmo proporre all!attenzione è Ugo di San Vittore (†1141), il grande maestro dell'abbazia parigina fondata da Guglielmo di Champeaux e divenuta un centro di vita spirituale e al tempo stesso di studi filosofici, aperti anche a quell'arte dialettica che aveva suscitato l'opposizione di Bernardo di Chiaravalle. Ugo compose fra l'altro un trattato De tribus diebus, le cui pagine iniziali esprimono in maniera esemplare una visione del rapporto uomomondo assai diffusa nel cosiddetto medioevo: "Tutto questo mondo sensibile è infatti come un libro scritto dalle mani di Dio, cioè creato dalla potenza divina, e le singole creature sono come figure, non inventate dall'arbitrio dell'uomo, ma istituite dalla volontà di Dio per manifestare ed indicare la sua invisibile sapien$\mathrm{za}^{3}$.

La metafora del gran libro della natura, destinata a godere di vasta fortuna ben oltre il secolo XII, ${ }^{4}$ è a tutti noi familiare, ed è evidente l'ispirazione agostiniana e, prima ancora, paolina del discorso di Ugo, che non a caso si apre con la citazione della Lettera ai Romani, 1, 20 (Invisibilia Dei a creatura mundi per

dimostrazione della sussistenza dell'anima. Ma si veda, della stessa autrice, $A$ proposito di uomo e natura nel secolo XII, "Filosofia", 18 (1967), p. 295-308, rist. in id., Studi di filosofia medievale, Milano 1978, p. 248-263. Per un ampio ventaglio di approcci al tema generale cfr. Mensch und Natur im Mittelalter, hrsg. von A. Zimmermann und A. Speer, Berlin-New York 1991 (Miscellanea Mediaevalia, 21), tomi 2. Fra i contributi più recenti segnaliamo quello di H. O. BIZZARRI, Una disputa entre filósofos y téologos: la concepción de la "naturaleza" en las colecciones sapienciales castellanas, "Medioevo", 22 (1996), p. 303-334.

3 "Universus enim mundus iste sensibilis quasi quidam liber est scriptus digito Dei, hoc est virtute divina creatus, et singulae creaturae quasi figurae quaedam sunt non humano placito inventae, sed divino arbitrio institutae ad manifestandam et quasi quodammodo significandam invisibilem Dei sapientiam" (UGO di SAN VTTTORE, I tre giomi dell'invisibile luce. L'unione del corpo e dello spirito, introduzione, testi emendati, traduzioni e note a cura di V. Liccaro, Firenze 1974, § 4, p. 5657). Sulla posizione speculativa espressa da Ugo in questo trattato si veda l'ampia "Introduzione", ibi, p. 17-45; v. inoltre V. LICCARO, L'uomo e la natura nel pensiero di Ugo da San Vittore, in La filosofia della natura nel medioevo, cit., p. 305-313; ID., Studi sulla visione del mondo di Ugo di S. Vittore, Udine 1969. Cfr. pure R. BARON, L'idée de nature chez Hugues de Saint Victor, in La filosofia della natura nel medioevo, cit., p. 260-263.

4 Cfr. E. GARIN, La nuova scienza e il simbolo del libro, "Rivista critica di storia della filosofia", 29 (1974), p. 328-334. 
ea quae facta sunt intellecta conspiciuntur)..$^{5}$ In una mirabile pagina delle Confessioni s. Agostino aveva fornito una chiave di lettura del mondo: l'uomo si volge attorno e si chiede chi sia Dio, ma il mare e gli abissi e gli animali e gli astri del cielo rispondo concordi "Non siamo noi Dio; egli, anzi, ci ha creato". Queste risposte ci sono portate, "a guisa di messaggeri", dại nostri sensi, che rinviano all'"uomo interiore", di cui essi sono solo lo strumento (Homo interior cognovit haec per exterioris ministerium). ${ }^{6}$ Anche gli animali vedono la bellezza (species) del mondo, ma non sono in grado d'interrogarla sulla sua origine, "poiché ai sensi che danno loro indicazioni non è preposta, come giudice, la ragione (ratio). Gli uomini invece possono interrogarla, in modo da riuscire a vedere la invisibile perfezione di Dio, una volta che, attraverso le cose create, n'abbiano avuto cognizione. Ma essi si lasciano assoggettare dalla passione per le cose create (sed amore subduntur eis), e quando sono assoggettati più non sono capaci di farne giudizio".

Fedele a questa impostazione del rapporto uomo-natura-Dio, Ugo di San Vittore distingue nettamente fra l'atteggiamento dell'insipiens e quello del sapiens. Come l'analfabeta (illetteratus) non sa decifrare una pagina scritta, così l'“animalis homo, qui non percipit ea quae Dei sunt (1 Cor 2, 14)", si limita a guardare la bellezza esteriore delle creature, sed intus non intelligit rationem, non riesce cioè a cogliere nelle creature il segno della "mirabile sapienza del creatore". Ed ecco l'atteggiamento di contemplazione che il sapiens deve assumere di fronte alla natura in quanto opera di Dio: "Eे bene dunque contemplare ed ammirare assiduamente le opere divine, ma da parte di coloro che sanno volgere ad un fine spirituale (in usum spiritualem) la bellezza (pulchritudinem) delle realtà corporee. Infatti proprio per questo motivo la Sacra Scrittura ci invita cosi insistentemente a considerare le mirabili realtà create da Dio, affinché, per mezzo delle cose che vediamo nel mondo esterno, possiamo giungere nel nostro intimo alla conoscenza della verità (ut per ea quae foris credimus intus ad agnitionem veritatis veniamus)". ${ }^{8}$ La contemplatio et admiratio, dunque, come modalità di lettura della "mole del mondo". Ma se la bellezza della natura ci rinvia alla sapienza del creatore, "l'immensa grandezza delle creature rivela la potenza divina", mentre "la loro utilità manifesta la sua benignità". ${ }^{9}$ Il Leitmotiv agostiniano, ritmato sul nesso trinitario, fornisce dunque l'impianto generale al De tribus diebus, ma in questa sede ci preme rilevare come il rapporto uomo-natura non si risolva nel riconoscimento della potenza e sapienza di Dio, ma presenti anche il tema della utilitas del creato, segno della benignitas del creatore verso quella speciale creatura che è l'uomo.

Già trattando della dispositio rerum, che suscita ammirazione in chi osservi con diligenza la natura, Ugo aveva tracciato un ampio affresco del mondo, tutto ispirato a una visione finalistica: gli astri sono stati collocati in cielo per poter illuminare "tutte le realtà poste sulla terra"; la disposizione delle nuvole è tale da

UGO di SAN VITTORE, I tre giomi, cit., § 1, p. 48.

AUG. Conf. 10, 6, 9 (SANT'AGOSTINO, Le Confessioni, introd, testo e traduzione a cura di A.

Marzullo, Bologna 1968, p. 598-599).

7 AUG. Conf. 10, 6, 10 (tr. Marzullo, p. 600-601).

UGO di SAN VITTORE, I tre giomi, cit., § 4, p. 56-59.

9 Ibi, § 1, p. 48-49. 
far cadere la pioggia sulla superficie terrestre, nel cui grembo si raccolgono grande masse d'acqua; gli animali sono armonicamente distribuiti nell'aria, nell'acqua e sulla terra; la varietà geografica garantisce una varietà di prodotti naturali e di attività umane... In particolare la provvidenza del creatore ha fatto sì che siano diffuse e facilmente accessibili "quelle cose che sono necessarie ai bisogni dell'uomo (ea quae humanis usibus necessaria sunt)", laddove "quelle realtà che sono oggetto della ricerca non per naturale necessità, ma per brama di possesso, a motivo della loro bella apparenza, sono state nascoste da Dio nelle occulte viscere della terra, affinché sia vinto almeno dal fastidio della fatica, e così trovi modo di stare tranquillo, colui che l'amore della virtù non aveva trattenuto dallo smoderato desiderio (immoderato appetitu)" ${ }^{10}$

Alla utilitas rerum (che è il risultato, lo ricordiamo, della benignitas di Dio) Ugo dedica in maniera specifica il § 14 , ove si distinguono "quattro aspetti: il necessario, il comodo, l'adatto e il gradito (necessaria, commoda, congrua et grata)". I primi due corrispondono al significato ancor oggi corrente, mentre "adatto e conveniente è ciò che, pur non portando un diretto vantaggio a chi ne usa, tuttavia ben s'addice a chi se ne serve", come nel caso delle tinture coloranti per gli abiti o delle gemme preziose; invece "gradito è ciò che pur non essendo di alcuna utilità, piace a vedersi: sono tali forse alcune specie di animali e di vegetali, di uccelli e di pesci". Ma per quale ragione - si chiede Ugo - Dio ha creato tutte queste cose che non sono strettamente necessarie alla vita dell'uomo? In termini più moderni: perché questa sovrabbondanza nel creato, ossia nella natura, che parrebbe andar contro il principio di economia? L'articolata risposta merita d'essere riprodotta per esteso, perché sintetizza la visione vittorina del rapporto (Dio)-uomo-mondo:

Dio ha fatto l'uomo per sé (propter se) e tutte le altre cose per l'uomo (propter hominem). Ha fatto l'uomo per sé, non perché egli avesse bisogno dell'uomo, ma per offrirgli la possibilità - della quale migliore non poteva offrirsi - di godere di Dio stesso; ha creato tutte le altre cose affinché fossero per destinazione sottoposte all'uomo e servissero alla sua utilità (alia vero creatura sic facta est, ut et subiecta homini esset per conditionem, et deserviret ad utilitatem). L'uomo dunque, collocato per cosi dire in posizione mediana (in quodam medio), ha sopra di sé Iddio e sotto di sé il mondo; riguardo al suo corpo è unito al mondo verso il basso, riguardo al suo spirito è elevato verso l'alto, cioè verso Dio. Fu necessario, dunque, che la creazione delle realtà visibili fosse ordinata in modo tale, che l'uomo potesse riconoscere in esse, esternamente a sé, un segno di quel bene invisibile, che doveva cercare dentro di sé [...]. Non conveniva dunque che la ricchezza delle realtà visibili (renum visibilium copia) patisse difetto in alcuna parte, poiché è stata creata soprattutto al fine di annunciare l'inestimabile abbondanza dei beni eterni. Ecco così la risposta al quesito perché Dio volle creare anche quelle realtà che sapeva essere non necessarie all'uomo. Se egli infatti avesse donato soltanto le cose necessarie, sarebbe stato buono, ma non generoso (dives); poiché invece ha aggiunto alle cose necessarie anche quelle comode, ha mostrato

${ }^{10} \mathrm{Ibi}, \S 5, \mathrm{pp} .66-67$. Questo tema, riferito ai metalli rari e alle pietre preziose, verrà ripreso più avanti (\$10, p. 78-81). 
le ricchezze della sua bontà. Poiché, ancora, oltre alle cose comode ha aggiunto quelle che sono convenienti, ha rivelato le grandi ricchezze della sua bontà. Infine quando alle realtà convenienti ha aggiunto nel dono anche quelle che sono gradite e piacevoli, che altro avviene se non la manifestazione della somma e sovrabbondante ricchezza della sua generosità (quid aliud quam superabundantes divitias bonitatis suae notas facit)?

Il messaggio che si ricava da queste pagine di Ugo di San Vittore è lineare: nella grandezza e bellezza della natura l'uomo contempla con ammirazione i segni della divina potenza e sapienza, facendo da tramite fra il creatore e le multiformi realtà terrene; queste ultime sono, per volontà e bontà di Dio, al servizio dell'uomo. Nell'immaginario medievale (si pensi alle miniature che illustrano il Genesi, oppure agli splendidi mosaici della prima metà del sec. XIII che ornano la cupola della prima campata del nartece nella basilica di San Marco a Venezia) ${ }^{12}$ il dominio che l'uomo esercita sulla natura era simboleggiato dall'atto con cui Dio fa sfilare gli animali davanti ad Adamo, che impone loro il nome; e l'impositio nominum è al tempo stesso una presa di possesso (o meglio un affidamento, giacché anche Adamo è creatura di Dio) e un atto di conoscenza, in quanto si riteneva che il linguaggio del nostro progenitore, grazie alla diretta illuminazione divina, fosse in grado di cogliere la realtà intima e costitutiva degli esseri. ${ }^{13}$

E veniamo all'altro autore, che non fu certo un filosofo in senso professionale e neppure un teologo, anche se le sue prose abbondano di riflessioni filosoficomorali: Francesco Petrarca. Họmo novus per eccellenza, proiettato ormai in una dimensione "moderna", il Petrarca innovò anche nel modo d'intendere il rapporto con la natura, com'ebbe a rilevare Ernst Cassirer in alcune pagine di grande efficacia di Individuum und Cosmos (1927), un'opera che ha fortemente segnato l'interpretazione dei rapporti fra medioevo ed "età nuova" nel corso del XX secolo. Tre sono gli aspetti della svolta che il cantore di Laura impresse al modo di concepire il "senso della natura". Anzitutto la sua poesia lirica "è la prima che tolga la natura dal confino al quale l'aveva condannata la concezione dogmaticomedioevale. Essa si libera ora da tutto ciò che conteneva di estraneo, di demoniaco, d'inquietante, ché l'ispirazione lirica non va a cercare in essa ciò che ha di antitetico alla realtà psichica, ma ritrova in lei le tracce e l'eco dell'anima". ${ }^{14}$

Per tale via - ed ecco il secondo aspetto - "il paesaggio diviene per il Petrarca lo specchio vivente dell'anima". La natura è descritta e contemplata non per se

11 Jbi, § 14, p. 90-93.

12 Cfr. in proposito X. MURATOVA, "Adam donne leurs noms aux animaux". L'iconographie de la scène dans l'art du Moyen Age: les manuscrits des bestiaires enluminés du XIle et du XIIle siècles, "Studi medievali", XVIII/2 (1977), p. 367-394.

13 Su questa "teologia del linguaggio", che trae origine dai commentari biblici di Filone di Alessandria, cfr. A. BORST, Der Turmbau von Babel. Geschichte der Meinungen über Ursprung und Vielfalt der Sprachen und_Völker, Stuttgart 1957-1963, I, pp. 119-120, 147-148, 170-171, 250-251; I/1, pp. 392 e 478. Ma si veda pure, sul "dominio" concesso ad Adamo, R. LAMBERTINI, La proprietà d'Adamo. Stato d'innocenza ed origine del "dominium" nel Commento alle Sentenze e nell'Improbacio" di Francesco d'Ascoli, "Bullettino dell'Istituto storico italiano per il medio evo ed Archivio Muratoriano", $\mathrm{n}^{\circ}$ 99/2 (1994), p. 201-252.

14 E. CASSIRER, Individuo e cosmo nella filosofia del Rinascimento, tr. it., Firenze 1967, pp. 227-228. 
stessa 0 in quanto vestigia del creatore, ma in quanto "nuovo mezzo" per ricondurci alla contemplazione del nostro io. Da qui deriva il terzo aspetto: il senso della natura risulta di fatto limitato e compresso, giacché, "in questa sua funzione di riflettere l'elemento psichico, la natura non ha che una realtà mediata e, per così dire, riflessa". Questo ripiegamento interiore, che è condizione per "l'unico rapporto vero ed immediato" (ossia, agostinianamente, "il rapporto dell'anima con Dio"), finisce così coll'impedire "una comunione diretta con la natura, col mondo dell'intuizione esteriore". Il celebre episodio della faticosa ascesa al Mont Ventoux (Familiares, IV, 1), quando il poeta, giunto alfine sulla vetta, non si volge a contemplare la natura ma si china sul libro che aveva portato seco, rende in maniera emblematica questo atteggiamento tutto interiore, che guarda alla natura come a un semplice sfondo. ${ }^{15}$

Non è certo il caso d'insistere sull'inadeguatezza interpretativa del primo aspetto (la "liberazione" della natura da una visione dogmatica e demoniaca), il quale risente fortemente del fascinoso cliché burckhardtiano e denota una sostanziale incomprensione della cultura medievale. I passi sopra citati del De tribus diebus di Ugo di San Vittore inducono semmai a una valutazione opposta, che vede la natura esaltata e idealizzata quale vero e proprio "libro" della creazione; e ciò richiamandosi allo stesso maestro (s. Agostino) al quale s'ispira, con sensibilità assai diversa, anche il Petrarca... Giova piuttosto evidenziare gli altri due aspetti, da cui si evince che la natura è stata creata per 'parlare' in primis non tanto del creatore quanto dell'uomo stesso, in una specularità che valorizza la sfera del sentimento e vede il mondo come una tela di fondo su cui si proietta l'umana soggettività: una sorta di antropizzazione, dunque, di taluni elementi della natura, cui fa pendant la riduzione ad 'oggetto' di tutti gli altri elementi che non siano funzionali al sentire del soggetto uomo.

Si obbietterà, a questo punto, che quella del Petrarca è una testimonianza di grande interesse e tuttavia troppo personale ed isolata per assurgere a indice rappresentativo di una generale mutazione - sul finire dell'età medievale - nel modo di guardare alla natura. $\mathrm{Ma}$, a ben vedere, è lo stesso Petrarca a segnalarci tale avvenuta mutazione, sia pure in termini critici e quindi tendenzialmente deformanti, che vengono comunque a combaciare con la prospettiva sopra ricordata del rapporto uomo-natura, integrandola con l'aspetto più propriamente filosofico-scientifico. Rileggiamo, ad esempio, le pagine iniziali del De sui ipsius et multorum ignorantia, in cui il Petrarca si scaglia contro alcuni suoi amici che erano soliti proporgli "o qualche problema di filosofia aristotelica o qualche argomento di zoologia", e sdegnarsi se egli rifiutava l'autorità di Aristotele, "come se da filosofi ed amatori della sapienza [sapientiae studiosis amatoribus: è evidente l'impronta agostiniana] fossimo tutti divenuti aristotelici o meglio pitagorici", legati all'ipse dixit. ${ }^{16}$ Ed è tipica l'insofferenza che il poeta manifesta per tale cultura, giudicata

${ }_{16}^{15}$ Ibi, p. 228-229.

16 F. PETRARCA, Prose, a cura di G. Martellotti e di P. G. Ricci, E. Carrara e E. Bianchi, MilanoNapoli 1955, p. 718-719. 
per lo più uno strumento di pazzia e di vana superbia che ci distoglie da ciò che per l'uomo vale veramente:

"Quel tale sa una quantità di cose sugli animali feroci, sugli uccelli, sui pesci: quanti peli ha il leone sulla testa, quante piume l'avvoltoio sulla coda, con quante spire il polipo abbraccia il naufrago; [...] sa che il parto dell'orsa è deforme, raro quello della mula; unico e infelice quello della vipera; che le talpe sono cieche e le api sorde, che finalmente - di tutti gli esseri animati soltanto il coccodrillo è capace di muovere la mandibola superiore. Tutte cose false in grandissima parte [...]. Comunque, anche se fossero vere non servirebbero affatto a vivere felici. Di grazia, che può giovare conoscere belve, uccelli, pesci, serpenti, e ignorare ovvero non curarsi dell'uomo: ignorare lo scopo della vita, donde veniamo, dove andiamo (et naturam hominum, ad quid nati sumus, unde et quo pergimus, vel nescire vel spernere)?"

Le curiosità zoologiche qui sciorinate dal Petrarca appartengono a una consolidata tradizione, di cui - significativamente - si trova l'eco anche nel $D e$ tribus diebus di Ugo di San Vittore, in un contesto però assai diverso: al posto del fastidio per gl'inutili dettagli sul mondo naturale (oggetto di studio da parte degli "aristotelici") domina qui l'estatica contemplazione della potenza divina, che si manifesta non solo nell'atto di creare dal nulla, ma anche nella capacità di produrre cose innumerevoli e straordinarie. "Prova a contare - osserva Ugo con ritmo incalzante - le stelle del cielo, la sabbia del mare, i granelli di polvere, le gocce di pioggia, le penne dei volatili, le squame dei pesci, i peli degli animali, le erbe dei campi, le foglie ovvero i frutti degli alberi, e conta l'incalcolabile numero (innumerabilia numera) di tante altre incalcolabili cose" ${ }^{17}$ E più avanti, a proposito dell'ordinata bellezza delle realtà naturali da cui traspare la sapienza divina, Ugo esprime il suo ammirato stupore anche per le realtà che ai nostri occhi appaiono "strane o ridicole" (monstruosa vel ridicula); ed ecco rispuntare, insieme con altre meraviglie della natura, la particolarità anatomica del coccodrillo cui farà riferimento anche il Petrarca:

Come mai il coccodrillo mangiando non muove la mandibola inferiore? Come mai la salamandra rimane illesa nel fuoco? Chi ha dato al riccio gli aculei e gli ha insegnato ad avvoltolarsi tra i pomi caduti per il vento, in modo da caricarsene, si che poi si muove stridendo come un carro? E la formica, che nella previsione dell'inverno futuro riempie i suoi magazzini di piccoli grani? E il ragno, che tesse con la materia delle sue viscere i laccio per irretire la preda? Queste realtà testimoniano la sapienza di Dio (isti sunt testes sapientiae Dei). ${ }^{18}$

E' il momento di tirare le fila del nostro discorso, che sembra essersi paradossalmente ridotto a prendere atto di un modo differente - nel Petrarca e in Ugo di San Vittore - di guardare ai peli dei mammiferi e alle mandibole dei coccodrilli... L'astiosa polemica del Petrarca contro quelli che egli chiama genericamente "aristotelici" o "averroisti" riflette in realtà la profonda mutazione culturale determinata nell'Occidente latino dall' "ingresso" del corpus aristotelico e

UGO di SAN VITTORE, I tre giomi, cit., § 2, p. 52-53.

Ibi, § 11, p. 80-81. 
in particolare delle opere fisiche e biologiche dello Stagirita, e dalla successiva evoluzione che ha portato autori come Pietro d'Abano a privilegiare lo studio delle scienze naturali, giudicato più certo e sicuro delle scienze per eccellenza speculative, come la metafisica e la teologia: il che significava spostare l'interesse dalla causa prima alle cause seconde, ovvero agli enti particolari e concreti che pullulano nel mondo sublunare e suscitano la curiosità dei dotti. ${ }^{19}$

Difatti la molteplicità degli enti naturali non è più "letta" come un'immagine ontologica della trinità divina, ovvero come un rinvio ad "Altro", ma diventa un insieme di "cose" indagate e classificate con criteri puramente naturali. D'altro canto anche chi, come il Petrarca, non s'interessa di queste indagini vede gli enti naturali come "cose" a cui guardare con noncuranza (l'aura mistica che in Ugo avvolge gli attributi degli animali è totalmente svanita nel cantore di Laura...), a meno che la natura non serva da cornice o da specchio per l'espressione dei sentimenti personali: un atto in ogni caso mutevole, così come mutevole è la nostra vita affettiva, e rispetto al quale la natura finisce col diventare ancora una "cosa", uno sfondo che solo di riflesso viene ad assumere per noi (e grazie a noi) un senso.

Inutile sottolineare come questo processo di reificazione della natura porti con sé un'accentuazione dell'idea di "dominio" dell'uomo sulla natura stessa. Tale idea è direttamente proporzionale al venir meno dell'atteggiamento di contemplazione del creato, legato alla consapevolezza ch'esso è insondabile nella sua mirabile varietà di aspetti e fenomeni. A partire dal XIII secolo questa varietà, di fronte alla quale un Ugo di San Vittore non poteva far altro che esprimere la sua ammirazione, viene fatta oggetto d'indagine specifica, per svelarne i segreti e per porre tali conoscenze al servizio degli uomini, dando corpo alle profetiche istanze e aspettative di un Ruggero Bacone. II nesso sapere-potere sarebbe stato posto al centro della visione filosofica dell'altro Bacone, Francesco, e avrebbe caratterizzato l'approccio dei moderni ad una natura intesa come un gigantesco meccanismo di forze e di leggi, di cui è possibile giungere gradualmente a conoscenza onde volgerle all'umano progresso, mentre l'atto contemplativo è confinato nell'ambito estetico-sentimentale, ove domina un totale soggettivismo.

In apparenza irreversibile, questo trend culturale è entrato in crisi quando ci si è accorti che il dominio totale sulla natura, ossia lo sfruttamento indiscriminato delle risorse, mette a rischio la futura sopravvivenza dell'umanità, e che la niduzione della natura a cosa comporta, alla lunga, un'analoga reificazione dell'uomo entro lo schema produzione-consumo-riciclaggio, che tende a ridurre tutto - idealità comprese - a merce e quindi a "cosa". Pur nella varietà e ambiguità delle sue componenti, l'odierna coscienza ecologistica ha richiamato l'attenzione sulla necessità di rivedere a fondo talune idee-guida di quella che si è soliti chiamare "età baconiana", ed è in tale contesto che una riflessione sul modo in cui molti autori medievali - sulla scia di s. Agostino - concepivano il rapporto uomo-natura si rivela meno ingenua o patetica di quanto possa apparire a prima vista. Ciò che spicca in quegli autori - lo si è visto in Ugo di San Vittore - è il senso della creaturalità, il riconoscimento che il mondo non è solo un oggetto, una

Cfr. E. PASCHETTO, Pietro d'Abano medico e filosofo, Firenze 1984, p. 93-98. 
deiezione di cui l'uomo dispone a piacimento, ma una realtà il cui valore intrinseco ci rimanda a un Altro. Una realtà di cui fa parte anche l'uomo, seppure in una posizione privilegiata per le sua capacità razionali, e che rende quindi illecito un rapporto di totale sfruttamento, come se la natura fosse un'assoluta alterità (una "cosa", per l'appunto) da asservire alla pretesa umana di dominio.

Da questo punto di vista è ancor più essenziale, e quindi più efficace, il messaggio che ci viene da un testo estraneo al linguaggio filosofico e teologico, ma che nella sua assoluta semplicità e trasparenza costituisce per tutti noi, che ci apriamo a un nuovo millennio, una provocazione formidabile: è il Cantico di frate Sole, in cui il senso della creaturalità giunge al suo vertice, sino ad abbracciare, insieme con gli astri e i quattro elementi di questo mondo (aria, acqua, fuoco, terra) anche "sora nostra morte corporale", in un coro a più voci che nella comune lode al creatore trova un legame di unità fraterna e umile ("Laudate et benedicete mi' Signore et rengratiate/ e serviateli cum grande humilitate"). ${ }^{20}$ I senso della creaturalità ha dunque il suo corrispettivo nel senso della fraternità, che impedisce la totale reificazione degli "altri" (le diverse realtà naturali) ma anche di noi stessi. Ed è ancora il Cassirer che ha espresso con chiarezza questa prospettiva di san Francesco:

L'amore non è più rivolto solo a Dio, come alla sorgente ed all'origine trascendente dell'essere, e non rimane neppure limitato alle relazioni fra uomo e uomo, come rapporto morale immanente. Esso va a tutte le creature in quanto tali [...] [che] non sono più "parti" indipendenti ed isolate dell'essere, ma vengono fuse dall'ardore dell'amore mistico in un tutto con l'uomo e con Dio. La categoria specifica ed individuale dell'"essere cosa", che rende possibile alla vita della natura di suddividersi in specie ben determinate e di graduarsi secondo determinati gradi, non regge davanti alla categoria mistica della fraternità... ${ }^{21}$

Riusciremo, noi uomini disincantati di fine millennio, a fare nostro questo senso di creaturalità e di universale fratellanza? Ovverosia, in termini più stringenti, saremo capaci di riconsiderare criticamente il nesso fra conoscenza e dominio?

Poeti del Duecento, a cura di G. Contini, Milano-Napoli 1960, I, p. 33-34.

CASSIRER, Individuo e cosmo, cit., p. 88. 